\title{
Hippoboscidae (Diptera, Hippoboscoidea) no Estado do Paraná, Brasil: chaves de identificação, hospedeiros e distribuição geográfica ${ }^{1}$
}

\author{
Gustavo Graciolli ${ }^{2} \&$ Claudio José Barros de Carvalho ${ }^{2}$
}

\author{
${ }^{1}$ Contribuição número 1436 do Departamento de Zoologia, Universidade Federal do Paraná. \\ 2 Departamento de Zoologia, Universidade Federal do Paraná. Caixa Postal 19020, 81531-980 Curitiba, Paraná, Brasil. \\ Bolsista CNPq. E-mail: mingau@bio.ufpr.br, cjbcarva@ufpr.br
}

\begin{abstract}
Hippoboscidae (Diptera, Hippoboscoidea) in the State of Paraná, Brazil: keys, hosts and geographic distribution. A survey of the louse flies species (Diptera, Hippoboscidae) in the State of Paraná, Brazil was carried out. Keys to eight genera and 15 species found are given. The following species are recorded for the first time in Paraná: Lipoptena (Lipoptenella) guimaraesi Bequaert, 1957; Stipolmetopoda legtersi Bequaert, 1955; Icosta (Ornithopomus) Iatifacies Bequaert, 1955; Icosta (Ornithpomus) rufiventris (Bigot, 1885); Icosta (Ardmoeca) albipennis (Say, 1823) and Olfersia bisulcata Macquart, 1847. Baryphthengus ruficapillus (Vieillot, 1818) (Momotidae) and Ciccaba virgata Carbin, 1849 (Strigidae) are new host records for Ornithoica vicina (Walker, 1849) and I. (Ardmoeca) albipennis; Chiroxiphia caudata (Shaw, 1793) and Schiffornis virescens (Lafresnaye, 1838) (Pipridae) for Ornithoctona fusciventris (Wiedemann, 1830) and Gallus gallus domesticus (Linnaeus, 1758) (Phasianidae) for S. legtersi.
\end{abstract}

KEY WORDS. Artiodactyla, birds, host-parasite relationship, louse flies, taxonomy.

A família Hippoboscidae compreende cerca de 200 espécies distribuídas por 21 gêneros, em três subfamílias. É encontrada em todas as regiões biogeográficas, parasitando aves e algumas ordens de mamíferos (MAA 1989). Lutz et al. (1915) realizaram a sinopse dos hipoboscídeos encontrados no Brasil, disponibilizando uma chave de identificação para oito gêneros e diagnose de 14 espécies. Deste então, Bequaert $(1954,1955,1957)$ e MAA (1969) acrescentaram várias informações sobre a distribuição de espécies e a associação com hospedeiros. Atualmente são encontradas no Brasil 30 espécies contidas em 10 gêneros (BEQUAERT 1957). No entanto, ainda há pouca informação sobre a distribuição e os hospedeiros em todas as regiões do País. Neste trabalho, apresenta-se os resultados de um levantamento taxonômico das espécies de Hippoboscidae e de seus hospedeiros no Estado do Paraná, assim como chaves de identificação dos gêneros e das espécies encontrados na região.

\section{MATERIAL E MÉTODOS}

Para a realização deste trabalho, além dos dados de literatura, foram examinados espécimes preservados em via líquida e secos provenientes das seguintes instituições brasileiras: Coleção de Entomologia Padre Jesus Santiago Moure do Departamento de Zoologia, Universidade Federal do Paraná, Curitiba, Paraná (DZUP); Museu de História Natural Capão da Imbuia, Curitiba, Paraná (MHNCI).

A terminologia adotada para a morfologia de Hippoboscidae segue MAa \& Peterson (1987).
A classificação, e os nomes científicos e populares das espécies de aves foram utilizados segundo SICK (1997). Em relação à classificação e nomes científicos das espécies de Artiodactyla (Mammalia), segue-se Grubb (1993).

\section{RESULTADOS}

Foram encontradas 15 espécies de Hippoboscidae pertencentes a oito gêneros de Lipopteninae e Ornithocinae. Como hospedeiros foram identificados três espécies de Artiodactyla (Mammalia) e 21 espécies de aves (Tab. I). Entre as espécies de Hippoboscidae, seis são registradas pela primeira vez no Paraná.

\section{Chave de identificação para gêneros de Hippoboscidae no Paraná}

1. Espiráculo torácico grande e arredondado. Basitarso III com comprimento semelhante aos tarsômeros subseqüentes, exceto o último

... 2

1'. Espiráculo torácico pequeno e elíptico. Basitarso III tão ou mais longo do que os dois ou três tarsômeros subseqüentes somados

3

2. Ocelo ausente. Olho retangular e estreito (Fig.1). Asa reduzida a pequena calosidade. Halter ausente ......... Melophagus

2 '. Ocelo presente. Olho semicircular (Figs 2-3). Asa desenvolvida e caduciforme (Fig. 26). Halter presente .... Lipoptena

3. Seta vertical interna inserida em protuberância arredonda- 
Tabela I. Relação hospedeiro-hipoboscídeo no Estado do Paraná, Brasil.

\begin{tabular}{|c|c|}
\hline Hospedeiro & Hipoboscídeo \\
\hline \multicolumn{2}{|l|}{ Mammalia Artiodactyla } \\
\hline \multicolumn{2}{|l|}{ Cervidae } \\
\hline Mazama sp. & Lipoptena (Lipoptenella) mazamae \\
\hline Ozotoceros bezoarticus (Linnaeus, 1758) (veado-campeiro) & Lipoptena (Lipoptenella) guimaraesi \\
\hline \multicolumn{2}{|l|}{ Bovidae } \\
\hline Ovis aries Linnaeus, 1758 (ovelha doméstica) & Melophagus ovinus \\
\hline \multicolumn{2}{|l|}{ Aves } \\
\hline \multicolumn{2}{|l|}{ Accipitridae } \\
\hline Accipter striatus Vieillot, 1808 (gaviãozinho) & Ornithoctona erythrocephala \\
\hline Ictinia plumbea (Gmelin, 1788) (sovi) & Ornithoctona erythrocephala \\
\hline \multicolumn{2}{|l|}{ Cathartidae } \\
\hline Sarcoramphus papa Linnaeus, 1758 (urubu-rei) & Olfersia bisulcata \\
\hline \multicolumn{2}{|l|}{ Columbidae } \\
\hline Columba livia Gmelin, 1789 (pombo-doméstico) & Pseudolynchia canariensis \\
\hline Zenaida auriculata (Des Murs, 1847) (pomba-avoante) & Ornithoctona erythrocephala \\
\hline \multicolumn{2}{|l|}{ Emberezidae } \\
\hline Zonotrichia capensis (Statius Muller, 1776) (tico-tico) & Ornithoctona fusciventris \\
\hline \multicolumn{2}{|l|}{ Falconidae } \\
\hline Micrastor ruficollis (Vieillot, 1817) (gavião-caburé) & Ornithoica vicina, Ornithoctona erythrocephala \\
\hline Milvago chimachima (Vieillot, 1816) (gavião-carrapateiro) & Ornithoctona erythrocephala \\
\hline \multicolumn{2}{|l|}{ Fregatidae } \\
\hline Fregata magnificens Mathews, 1914 (tesourão) & Olfersia spinifera \\
\hline \multicolumn{2}{|l|}{ Furnariidae } \\
\hline Sclerurus scansor (Ménétriés, 1835) (vira-folhas) & Ornithoctona fusciventris \\
\hline Syndactila rufosuperciliata (Lafresnaye, 1832) (trepador-quiete) & Ornithoica vicina \\
\hline \multicolumn{2}{|l|}{ Momotidae } \\
\hline Baryphthengus ruficapillus (Vieillot, 1818) (juruva) & $\begin{array}{l}\text { Ornithoica vicina, Ornithoctona erythrocephala, Icosta (Ardmoeca) } \\
\text { latifacies }\end{array}$ \\
\hline \multicolumn{2}{|l|}{ Muscicapidae } \\
\hline Turdus rufiventris Pallas, 1776 (sabiá-laranjeira) & Ornithoica vicina \\
\hline \multicolumn{2}{|l|}{ Phasianidae } \\
\hline Gallus gallus domesticus (Linnaeus, 1758) (galo-doméstico) & Stilpometopa legtersi \\
\hline \multicolumn{2}{|l|}{ Pipridae } \\
\hline Chiroxiphia caudata (Shaw, 1793) (tangará, dançador) & Ornithoctona fusciventris \\
\hline Schiffornis virescens (Lafresnaye, 1838) (flautim) & Ornithoctona fusciventris \\
\hline \multicolumn{2}{|l|}{ Ramphastidae } \\
\hline Ramphastos discolorus Linnaeus, 1758 (tucano-de-bico-verde) & Icosta (Ornithopomus) angustifrons \\
\hline \multicolumn{2}{|l|}{ Strigidae } \\
\hline Ciccaba virgata Cassin, 1849 (coruja-do-mato) & Ornithoica vicina, Icosta (Ardmoeca) latifacies \\
\hline Otus choliba (Viellot, 1817) (corujinha-do-mato) & Icosta (Ornithopomus) americana \\
\hline Speotyto cunicularia Molina, 1782 (coruja-buraqueira) & Icosta (Ornithopomus) americana \\
\hline Strix hylophila Temmick, 1825 (coruja-listrada) & Icosta (Ornithopomus) americana, Icosta (Ornithopomus) rufiventris \\
\hline \multicolumn{2}{|l|}{ Thamnophilidae } \\
\hline Thamnophilus caerulescens Viellot, 1816 (choca-da-mata) & Ornithoctona fusciventris \\
\hline
\end{tabular}

Revista Brasileira de Zoologia 20 (4): 667-674, dezembro 2003 
da (Fig. 4). Asa com veia $\mathrm{R}_{4+5}$ sinuosa, convergente com $\mathrm{C}$ (Fig. 27). Garra tarsal inteira, parecendo bífida (Fig. 19) .. . Ornithoica

3'. Seta vertical interna não inserida em protuberância arredondada (Figs 5 a 9). Asa com veia $\mathrm{R}_{4+5}$ quase retilínea (Figs 28 a 30). Garra tarsal bífida, parecendo trífida (Fig. 18) . 4

4. Antena tão ou mais longa que o palpo foliáceo (Fig. 5) ... 5

4'. Antena mais curta que o palpo cuneiforme (Fig. 6) ......... 6

5. Ocelos ausentes. Espiráculo mesotorácico dorsal. Laterotergito do posnoto com setas. Escutelo sem setas discais e com um pente de setas longas na margem posterior do escutelo (Fig. 13) ................................................... Stipolmetopoda

5'. Ocelos presentes. Espiráculo mesotorácico látero-dorsal. Laterotergito do posnoto glabro. Escutelo com setas discais e sem setas na margem posterior (Fig. 12) ..... Ornithoctona

6. Processos ântero-laterais da face subparalelos e largos (Figs 8-9). Laterotergito do posnoto com setas finas e com processo digitiforme (Fig. 16) Olfersia

6'. Processos ântero-laterais da face divergentes e finos (Figs 67). Laterotergito do posnoto com uma fileira de setas espiniformes e sem processo digitiforme.

7

7. Asa somente com veia $\mathrm{r}-\mathrm{m}$ presente (Fig. 30). Margem posterior do escutelo truncada (Fig. 14). Metabasesterno com um processo espiniforme cobrindo a base da coxa (Fig. 31) Pseudolynchia

7'. Asa com veias r-m e dm-cu presentes (Figs 28-29). Margem posterior do escutelo convexa (Fig. 15). Metabasesterno sem um processo espiniforme Icosta

\section{Lipopteninae}

\section{Lipoptena Nitzsch, 1818}

No continente americano, há quatro espécies de Lipoptena (Lipoptenella) e Lipoptena (Lipoptena) cervi (Linnaeus, 1758), introduzida da Região Paleártica, registradas. Todas as espécies parasitam veados dos gêneros Odocoileus Rafinesque, 1832; Ozotoceros Ameghino, 1891; Mazama Rafinesque, 1817 e Pudu Gray, 1852 (Peterson \& MAA 1970). No Paraná, são encontradas duas espécies.

\section{Chave de identificação para espécies de Lipoptena no Paraná}

1. Três a quatro setas fronto-orbitais (Fig. 2). Tíbia II com três setas espiniformes apicais na face ventral, sendo a mediana mais longa que as outras (Fig. 22). Quatro primeiros tarsômeros da perna 2 com 2-1-1-3 setas espiniformes póstero-ventrais, respectivamente (Fig. 20). Conetivo abdominal dorsal piloso L. guimaraesi.

$1^{\prime}$. Uma ou duas setas fronto-orbitais (Fig. 3). Tíbia II com duas setas apicais na face ventral (Fig. 23). Quatros primeiros tarsômeros da perna 2 com 2-1-1-1 setas espiniformes póstero-ventrais, respectivamente (Fig. 21). Conetivo abdominal dorsal com setas esparsas .................. L. mazamae

\section{Lipoptena (Lipoptenella) guimaraesi Bequaert, 1957}

Distribuição geográfica. Brasil (Mato Grosso, Tocantins, Goiás e Paraná) e Uruguai.

Hospedeiro. Ozotoceros bezoarticus (Cervidae, Odocoileinae).
Material examinado. Paraná, Palmas, Fazenda Santa Rita, 28/I/1950 (9 machos e 7 fêmeas, hospedeiro Ozotoceros bezoarticus, MHNCI) R. Lang leg.

Discussão. Novo registro para o estado do Paraná. Lipoptena guimaraesi estava restrita a região do Cerrado nos estados do Mato Grosso, Tocantins e Goiás, Brasil Central (BEQUAERT 1957, Desiderio et al. 1999) e ao Uruguai (JACKSON 1987).

\section{Lipoptena (Lipoptenella) mazamae Rondani, 1878}

Distribuição geográfica. Estados Unidos da América, México, Guatemala, Honduras, Cuba, Panamá, Colômbia, Venezuela, Trinidad e Tobago, Guiana, Suriname, Brasil (Amazonas, Pará, Mato Grosso, São Paulo, Paraná, Santa Catarina e Rio Grande do Sul), Equador, Peru, Paraguai, Argentina e Uruguai.

Hospedeiros. Mazama americana (Erxleben, 1777), Mazama guazoupira (G. Fisher, 1814), Odocoileus virginianus (Zimmermann, 1780) (Cervidae, Odocoileinae).

Material examinado. Paraná: Céu Azul, 05/II/2002 (5 fêmeas, hospedeiro Mazama sp., DZUP), E.C. Guzzo leg.

Discussão. Anteriormente, MAA (1969) citou a ocorrência de L. mazamae no estado do Paraná, porém, sem informações adicionais sobre a identidade do hospedeiro.

\section{Melophagus Latreille, 1802}

Gênero com duas espécies, sendo uma cosmopolita, Melophagus ovinus, introduzida pelo homem juntamente com seu hospedeiro a ovelha doméstica (Ovis aries Linnaeus, 1758) e a outra, Melophagus rupicaprinus Rondani, 1879, restrita à Região Paleártica (BEQUAERT 1942).

\section{Melophagus ovinus (Linnaeus, 1758)}

Distribuição geográfica. Estados Unidos da América (incluindo Arquipélago do Havaí), México, Panamá, Colômbia, Venezuela, Equador, Brasil (Pará, Paraná, Santa Catarina e Rio Grande do Sul), Peru, Bolívia, Argentina, Chile, Uruguai e Reino Unido (Ilhas Falkland). Cosmopolita.

Hospedeiro. Ovis aries (Bovidae, Caprinae).

Material examinado. Paraná: Curitiba, sem data (6 machos e 20 fêmeas, DZUP), U.M.K. leg. São José dos Pinhais, sem data (1 fêmea, DZUP), B. Fernandes leg.

\section{Ornithocinae}

\section{Ornithoica Rondani, 1878}

O gênero Ornithoica compreende 24 espécies divididas em dois subgêneros (MaA 1969, Sinclair 1995). Apenas duas espécies são encontradas no continente americano, Ornithoica confluenta e O. vicina, sendo que esta última já havia sido registrada para o Paraná (MAA 1969).

\section{Ornithoica (Ornithoica) vicina (Walker, 1849)}

Distribuição geográfica. Canadá, Estados Unidos da América, México, Cuba, Jamaica, Guatemala, Costa Rica, Panamá, Colômbia, Venezuela, Guiana, Brasil (São Paulo, Paraná, Santa Catarina e Rio Grande do Sul), Equador, Peru, Bolívia, Paraguai e Chile.

Hospedeiros. Cerca de 86 gêneros, 25 famílias de 10 ordens de pássaros (MAA 1969).

Material examinado. Paraná: Curitiba, Parque Primave-

Revista Brasileira de Zoologia 20 (4): 667-674, dezembro 2003 

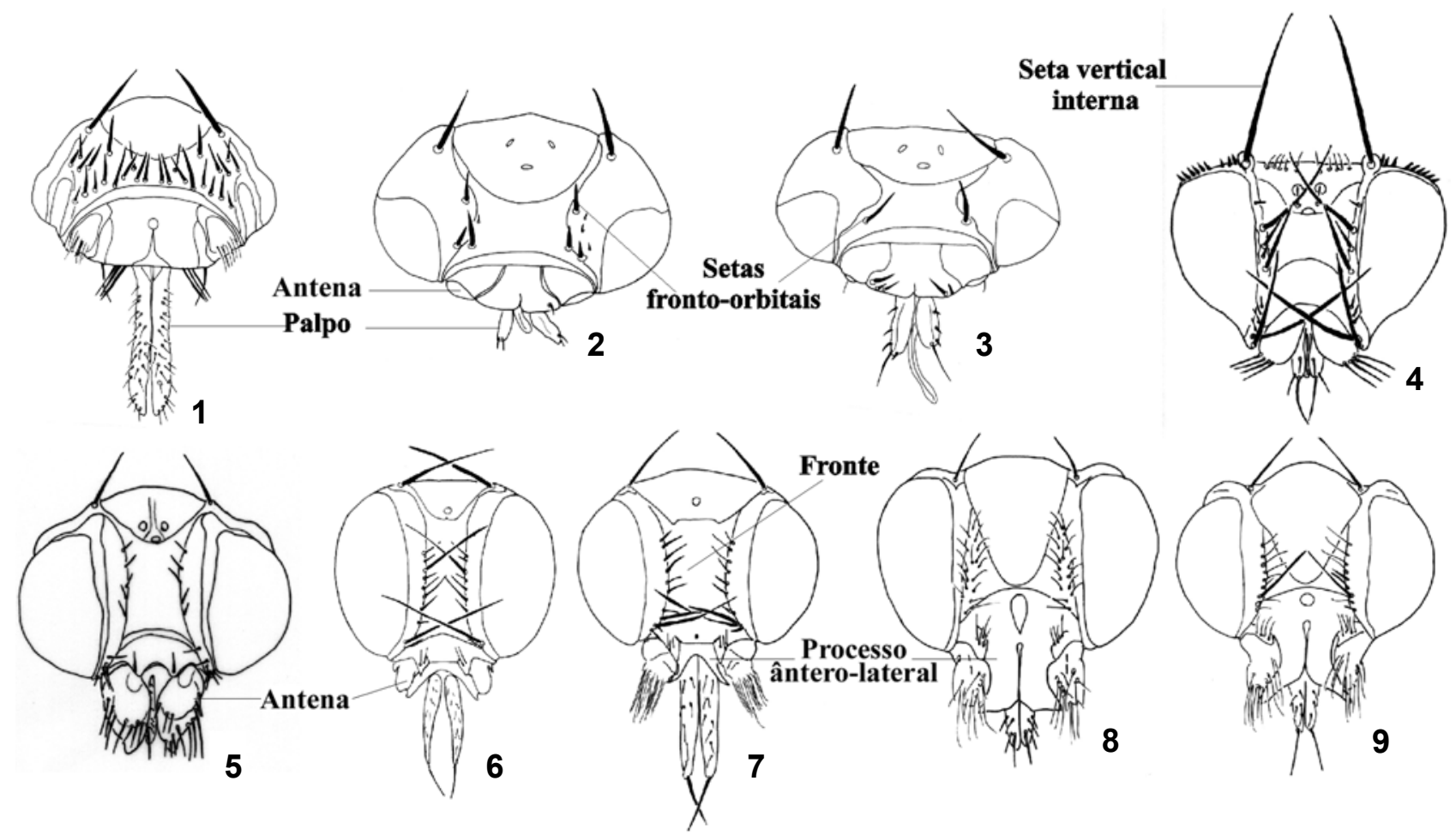

Figuras 1-9 Cabeça em vista dorsal. (1) Melphagus ovinus; (2) Lipoptena guimaraesi; (3) L. mazamae; (4) Ornithoica vicina; (5) Ornithoctona erythrocephala; (6) Icosta angustifrons; (7) I. latifacies; (8) Olfersia bisulcata; (9) O. spinifera. Figuras 6-7 e 8-9 adaptadas, respectivamente, de BequaERT $(1955,1957)$.

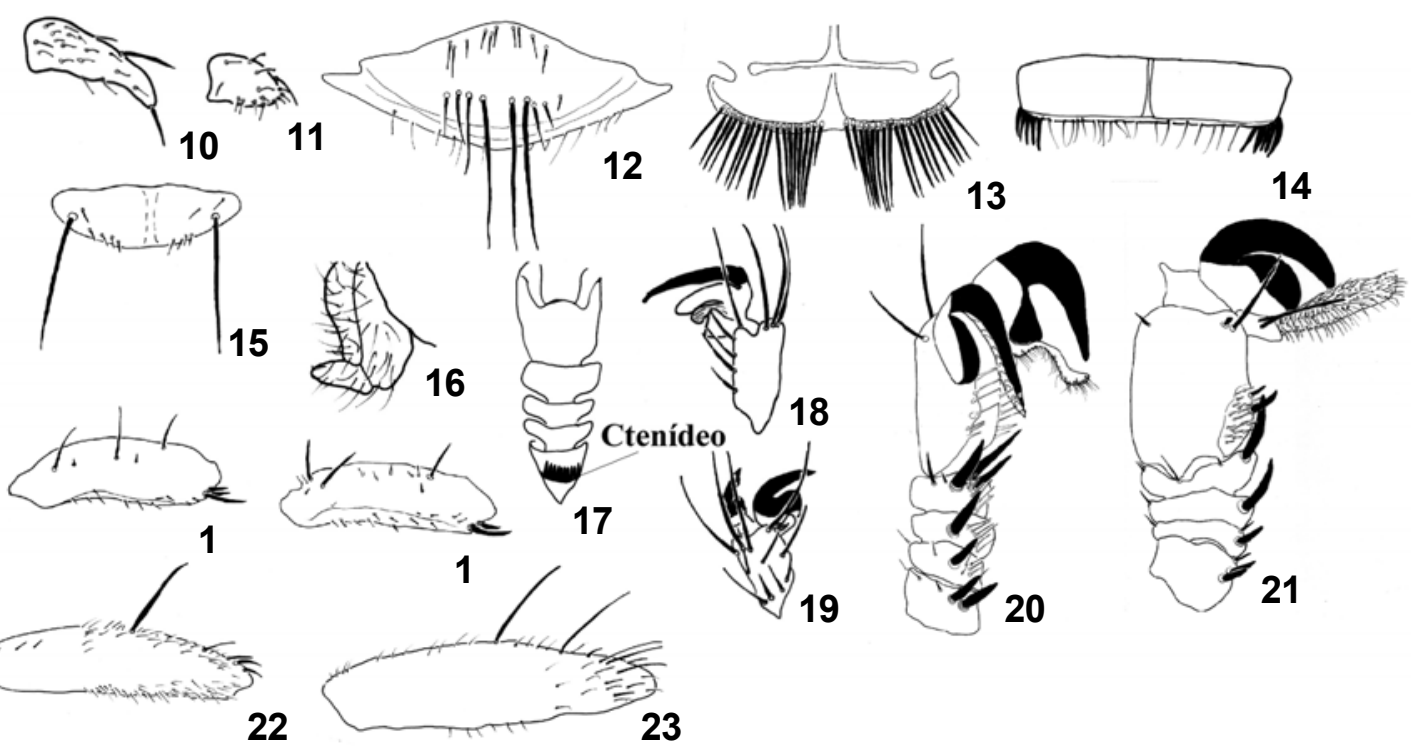

Figuras 10-25. (10) Palpo de Icosta angustifrons, vista lateral; (11) palpo de I. albipennis, vista lateral; (12) escutelo de Ornithoctona erythrocephala, vista dorsal; (13) escutelo de Stipolmetoba legtergi, vista dorsal; (14) escutelo de Pseudolynchia canariensis, vista dorsal; (15) escutelo de I. albipennis, vista dorsal; (16) laterotergito do posnoto de Olfersia bisulcata, vista dorsal; (17) tarso de Ornithoctona erythrocephala, setas e pretarso omitidos, vista ventral; (18) pretarso e último tarsômero de O. erythrocephala; (19) pretarso e último tarsômero de Ornithoica vicina; tarso II de (20) Lipoptena guimaraesi e de (21) L. mazamae; tíbia II de (22) L. guimaraesi e de (23) L. mazamae; (24) fêmur III de Icosta albipennis (Say), vista posterior e de (25) I. americana; vista posterior.

Revista Brasileira de Zoologia 20 (4): 667-674, dezembro 2003 
ra, 19/V/1995 (1 fêmea, hospedeiro Turdus rufiventris, MHNCI). Paranaguá, Floresta Estadual do Palmito, I/2003 (1 fêmea, hospedeiro Ciccaba virgata, MHNCI), E. Carrano leg. Piraquara, Mananciais da Serra, 06/VIII/2003 (1 fêmea, hospedeiro Syndactila rufoduperciliata, MHNCI), M.A. Marini leg. Três Barras do Paraná, Parque Estadual Rio Guarani (Margem direita do Rio Guarani), 07/V/2001 (1 macho, hospedeiro Baryphthengus ruficapillus, DZUP), M. Pichorin, A.M.K. Uejima, C. Gaho leg.

Discussão. Primeiro registro de O. vicina sobre $B$. ruficapillus e C. virgata. Anteriormente, BEQUAERT (1954) registrou $O$. vicina no Paraná sobre Micrastur ruficollis. Ornithoica vicina pode ser diferenciada de $O$. confluenta por apresentar ausência da microtríquias na célula 2a (Fig. 27).

\section{Ornithoctona Speiser, 1902}

Este gênero abriga 12 espécies, sendo cinco exclusivamente americanas. Apenas as espécies citadas na chave abaixo foram registradas no Paraná (MAA 1969).

\section{Chave de identificação para espécies de Ornithoctona no Paraná}

1. Asa glabra. Basitarsos com um ctenídeo ventral (Fig.17). Comprimento do corpo maior que $6 \mathrm{~mm}$...... O. erythrocephala

$1^{\prime}$. Asa com o terço apical parcialmente coberto por microtríquias. Somente o basitarso III com ctenídeo ventral. Comprimento do corpo menor que $5 \mathrm{~mm}$ O. fusciventris

\section{Ornithoctona erythrocephala (Leach, 1817)}

Distribuição geográfica. Canadá, Estados Unidos da América, México, Bahamas, Cuba, Jamaica, Ilhas Virgens, São Kitts e Nevis, Guadalupe, Dominica, São Vicente e Granadinas, Granada, Porto Rico, Guatemala, Nicarágua, Costa Rica, Panamá, Colômbia, Venezuela, Brasil (Mato Grosso, São Paulo, Paraná, Santa Catarina e Rio Grande do Sul), Equador, Peru, Bolívia, Argentina e Uruguai.

Hospedeiros. Cerca de 76 gêneros, 25 famílias de 14 ordens de aves (MAA 1969).

Material examinado. Paraná: Campo Mourão, VII/1989 (1 fêmea, hospedeiro Zenaida auriculata, DZUP), V. Moraes leg.; 22/IX/1989 (1 fêmea, hospedeiro Z. auriculata, DZUP), V. Moraes leg.; Fazenda Klabin, 17/X/1989 (1 fêmea, hospedeiro $Z$. auriculata, DZUP), V. Moraes leg.; Clevelândia, 27/XII/1982 (1 fêmea, DZUP), V.L. Camilotti leg.; Curitiba, São Braz, 14/III/ 2003 (4 fêmeas, hospedeiro Accipter striatus, MHNCI), L.F.F. de Macedo leg.; Foz do Iguaçu, XII/1967 (1 fêmea, DZUP), M.H.S. Bastos leg. Piraquara, Mananciais da Serra, 02/II/2003 (1 fêmea, hospedeiro Micrastor ruficollis, MHNCI), M.A. Marini leg. Segredo, 1991 (2 fêmeas, hospedeiro Ictinia plumbea, MHNCI); 1991 (2 fêmeas, hospedeiro Mivalgo chimachima, MHNCI). São José dos Pinhais, Roça Velha, 20/XI/2000 (1 fêmea, DZUP), A. de Meyer leg.; Telêmaco Borba, Parque Ecológico da Klabin, 16/XI/2000 (1 macho, DZUP), R.A. Machado leg.; Três Barras do Paraná, Parque Estadual Rio Guarani (Margem direita do Rio Guarani), 07/V/2001 (2 fêmeas, hospedeiro Baryphthengus ruficapillus, DZUP), M. Pichorin, A.M.K. Uejima, C. Gaho leg.

Discussão. Esta é a espécie com o maior número de exemplares nas coleções examinadas. Apresenta baixa especificidade parasitária. No Paraná, foi encontrada sobre quatro espécies de aves de quatro famílias. Ornithotocna erythrocephala foi registrada primeiramente no Paraná por BEQUAERT (1954).

\section{Ornithoctona fusciventris (Wiedemann, 1830)}

Distribuição geográfica. Canadá, Estados Unidos da América, México, Guatemala, Costa Rica, Panamá, Jamaica, Antilhas Maiores, Colômbia, Venezuela, Brasil (Minas Gerais, Rio de Janeiro, São Paulo, Paraná e Rio Grande do Sul), Equador, Peru, Chile e Argentina.

Hospedeiros. Cerca de 58 gêneros, 21 famílias de sete ordens de aves (MAA 1969).

Material examinado. Paraná: General Carneiro, 07/X/1990 (2 fêmeas, hospedeiro Zonotrichia capensis, DZUP), S.C. Luçolli leg. Piraquara, Mananciais da Serra (1000 metros), 13/I/2002 (1 macho, hospedeiro Chirexiphia caudata, DZUP), C.A.F.R. Gatto leg.

Discussão. Primeiro registro deste hipoboscídeo sobre $C$. caudata. No Paraná, O. fucisventris foi anteriormente registrada sobre Sclerus scansor e Thamnaphilus caerulescens (BEQUAERT 1954).

\section{Stipolmetopoda Coquillett, 1899}

Gênero exclusivamente americano com cinco espécies, das quais três foram registradas para o Brasil (MAA 1969).

\section{Stipolmetopoda legtersi Bequaert, 1955}

Distribuição geográfica. Brasil (Mato Grosso e Paraná) e Argentina.

Hospedeiros. Ortalis (Cracidae), Gallus gallus domesticus (Phasianidae).

Material examinado. Paraná: Fazenda Rio Grande, V/2002 (1 fêmea, hospedeiro Gallus gallus domesticus, DZUP), S.M. dos Santos leg.

Discussão. Novo registro para o estado do Paraná e primeira ocorrência deste hipoboscídeo sobre galo doméstico. Outras três espécies deste gênero, Stipolmetopoda impressa (Bigot, 1885), S. podopostyla Speiser, 1904 e S. fulvifrons (Walker, 1849), já tinham sido encontradas sobre fasianídeos domesticados (MaA 1969). Stipolmetopoda legtersi pode ser diferenciada das outras espécies do gênero por apresentar um par de setas verticais, processo metasternal longo sobre a coxa III e esternito VII mais longo que largo e com setas longas na margem posterior.

\section{Pseudolynchia Bequaert, 1926}

Gênero cosmopolita com cinco espécies reconhecidas. Apenas uma delas, Pseudolynchia brunnea (Latreille, 1812) é endêmica do continente americano e, por outro lado, P. canariensis é cosmopolita (MAA 1969).

\section{Pseudolynchia canariensis (Macquart, 1840)}

Distribuição geográfica. Estados Unidos da América, México, Cuba, Jamaica, Hispaniola, Porto Rico, Ilhas Virgens, São Kitts e Nevis, Antigua e Barbados, Honduras, Trinidad e Tobago, Colômbia, Venezuela, Guiana, Brasil (Amazonas, Ceará, Pernambuco, Minas Gerais, Rio de Janeiro, São Paulo, Paraná, Santa Catarina e Rio Grande do Sul), Argentina e Uruguai. Cosmopolita.

Hospedeiro. Columba livia (Columbidae).

Material examinado. Paraná: Curitiba, Centro, 17/XII/ 2000 ( 1 macho e 1 fêmea, DZUP), G. Graciolli leg. Londrina, 30/XI/2002 (1 macho, DZUP), E.C. Guzzo leg. Ponta Grossa, I/ 1949 (3 fêmeas, hospedeiro Columba livia, DZUP), C. Bruless leg.

Revista Brasileira de Zoologia 20 (4): 667-674, dezembro 2003 


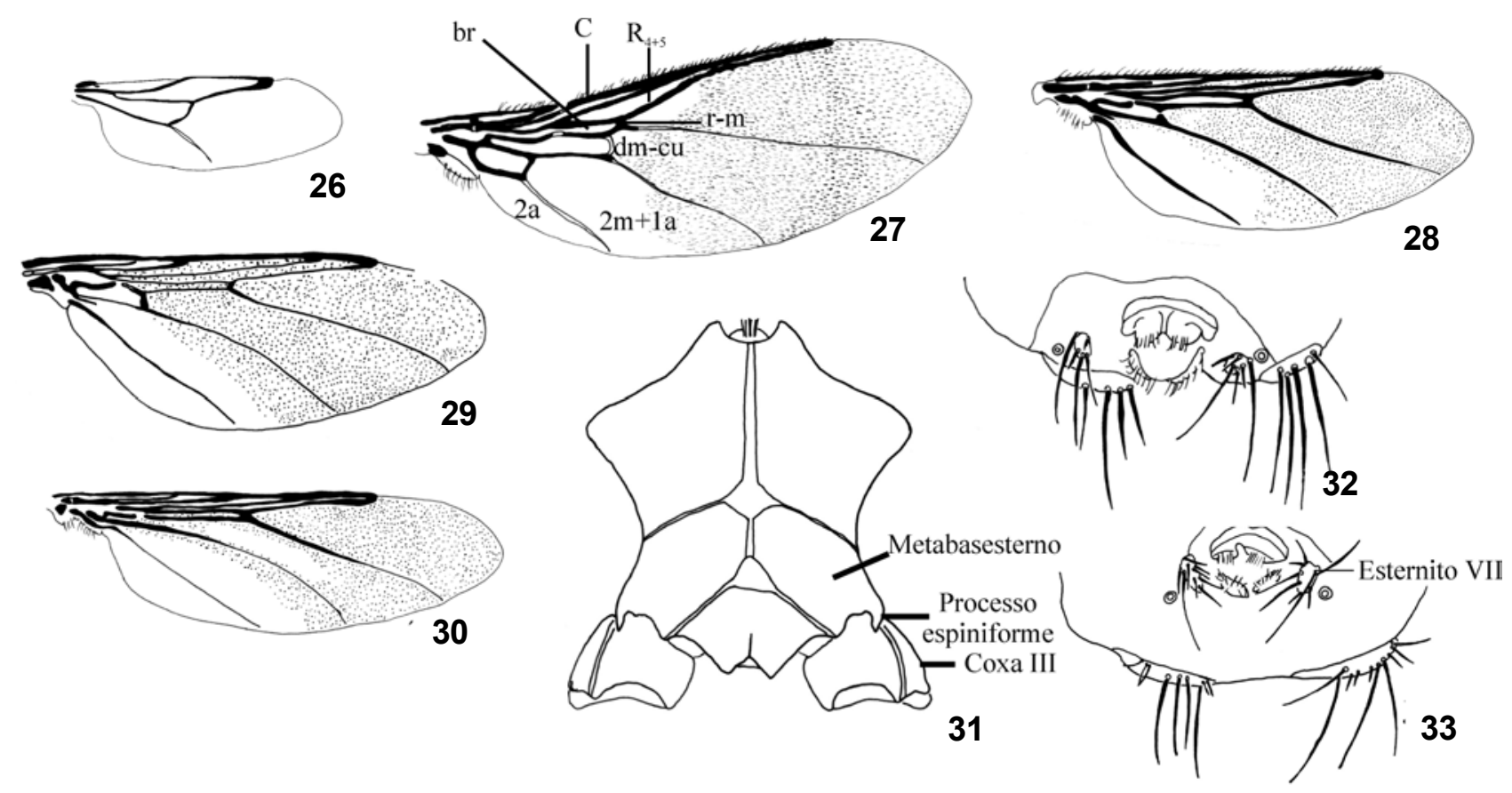

Figuras 26-33. (26-30) Asas: (26) Lipoptena sp., modificado de BequaERT (1942); (27) Ornithoica vicina; (28) Icosta latifacies; (29) I. rufiventris; adaptado de Bequaert (1955); (30) Pseudolynchia canariensis; (31) tórax de Pseudolynchia canariensis, vista ventral; ápice do abdômen da fêmea de (32) Icosta americana e de (33) I. rufiventris, em vista ventral.

Discussão. Provavelmente esta espécie foi introduzida no século XIX na América sobre seu hospedeiro, Columba livia (Bequaert 1955). Não há registro de P. canariensis parasitando aves nativas do continente americano. Pseudolynchia canariensis difere de $P$. brunnea pela ausência de microtríquias na maior parte da $2 \mathrm{~m}+1 \mathrm{a}$ (Fig. 30 ).

\section{Icosta Speiser, 1905}

Gênero cosmopolita, constituído por 45 espécies divididas em cinco subgêneros. No continente americano foram registradas 10 espécies em dois subgêneros, Ornithopomus Aldrich, 1923 e Ardmoeca Maa, 1963 (MAA 1969). No Paraná, foram registradas cinco espécies.

\section{Chave de identificação para espécies de Icosta no Paraná}

1. Palpo em vista lateral cerca de 1,5 a 2 vezes mais longo que alto (Fig. 11). Quatro primeiros tarsômeros do tarso I com 1-0-0-0 setas póstero-ventrais, respectivamente. Fêmur III com uma seta póstero-dorsal mais longa que a altura do fêmur (Fig. 24) I. (Ardmoeca) albipennis

$1^{\prime}$. Palpo em vista lateral além de 2 vezes mais longo que alto (Fig. 10). Quatro primeiros tarsômeros do tarso I com 2-11-0 setas póstero-ventrais, respectivamente. Fêmur III com no mínimo duas setas póstero-dorsais mais longas que a altura do fêmur (Fig. 25).......... I. (Ornithopomus) ............ 2

2. Palpo mais longo que a fronte (Fig. 7). Célula br glabra (Fig. 28) I (Ornithopomus) latifacies
2'. Palpo mais curto que a fronte (Fig. 6). Célula br totalmente ou parcialmente coberta por microtríquias (Fig. 29) ...... 3

3. Asa com 7 a $8,5 \mathrm{~mm}$ de comprimento. Lóbulo do sintergito I+II com quatro a seis setas espiniformes e laterito II com duas a quatro setas espiniformes

$3^{\prime}$. Asa com 5 a $6 \mathrm{~mm}$ de comprimento. Lóbulo do sintergito I+II com uma a duas setas espiniformes e laterito II sem setas espiniformes ............... I. (Ornithopomus) angustifrons

4. Esternito VII pelos menos duas vezes mais longo que largo (Fig. 33). Palpo em vista lateral, além de duas vezes mais longo que alto. Fêmur III com três setas longas dorsais ... I. (Ornithopomus) rufiventris

4'. Esternito VII menos de duas vezes mais longo que largo (Fig. 32). Palpo em vista lateral, duas vezes mais longo que alto. Fêmur III com quatro setas longas dorsais dispostas em duas filas.

I. (Ornithopomus) americana

\section{Icosta (Ardmoeca) albipennis (Say, 1823)}

Distribuição geográfica. Canadá, Estados Unidos da América, Bahamas, Cuba, Jamaica, Porto Rico, México, Honduras, El Salvador, Panamá, Colômbia, Brasil (Piauí, Mato Grosso, Minas Gerais, Goiás, Espírito Santo, São Paulo, Paraná e Rio Grande do Sul), Equador (incluindo Arquipélago de Galápagos), Paraguai e Argentina.

Hospedeiro. Ardeidae.

Material examinado. Paraná: XII/1950 (1 macho, hospedeiro socó, DZUP).

Discussão. Novo registro para o Paraná. 


\section{Icosta (Ornithoponus) americana (Leach, 1817)}

Distribuição geográfica. Canadá, Estados Unidos da América, México, Honduras, Costa Rica, Panamá, Colômbia, Venezuela, Guiana, Brasil (Goiás, Minas Gerais, São Paulo, Paraná, Santa Catarina e Rio Grande do Sul), Peru, Bolívia, Argentina.

Hospedeiros. 33 gêneros, 14 famílias de 8 ordens de aves (MAA 1969).

Material examinado. Paraná: Guarapuava, 17/X/2000 (2 fêmeas, hospedeiro Speotyto cunnicularia, DZUP), M.O. Moura leg.

Discussão. BEQUAERT (1955) já tinha registrado I. americana sobre Speotyto cunicullaria, Otus choliba e Strix hylophila no Paraná.

\section{Icosta (Ornithoponus) angustifrons (Wulp, 1903)}

Distribuição geográfica. Canadá, Estados Unidos da América, México, Honduras, Belize, Guatemala, Costa Rica, Panamá, Colômbia, Venezuela, Guiana e Brasil (Minas Gerais, Espírito Santo, Rio de Janeiro, São Paulo, Paraná, Santa Catarina e Rio Grande do Sul).

Hospedeiros. 29 gêneros, 12 famílias de 8 ordens de aves (MAA 1969).

Material examinado. Nenhum.

Discussão. Espécie anteriormente registrada no Paraná sobre Micrastor ruficollis (BEQUAERT 1955).

\section{Icosta (Ornithoponus) Iatifacies Bequaert, 1955}

Distribuição geográfica. Colômbia, Brasil (São Paulo e Paraná) e Paraguai.

Hospedeiros. Piaya cayana (Cuculidae), Baryphthengus ruficapillus (Momotidae) e Ciccaba virgata (Strigidae).

Material examinado. Paraná: Paranaguá, Floresta Estadual do Palmito, I/2003 (2 machos e 1 fêmea, hospedeiro Ciccaba virgata, MHNCI), E. Carrano leg. Três Barras do Paraná, Parque Estadual Rio Guarani (Margem direita do Rio Guarani), 07/V/2001 (1 macho, hospedeiro Baryphthengus ruficapillus, DZUP), M. Pichorin, A.M.K. Uejima, C. Gaho leg.

Discussão. Novo registro para o Paraná e nova ocorrência de hospedeiro, B. ruficapillus e C. virgata. Icosta latifacies foi encontrada junto de Ornithoica vicina sobre o mesmo indivíduo de B. ruficapillus e C. virgata.

\section{Icosta (Ornithoponus) rufiventris (Bigot, 1885)}

Distribuição geográfica. Estados Unidos da América, México, Costa Rica, Panamá, Venezuela, Guiana, Brasil (Paraná e Santa Catarina), Bolívia e Argentina.

Hospedeiros. 12 gêneros, três famílias de duas ordens (MAA 1969).

Material examinado. Paraná: Segredo, sem data (duas fêmeas, hospedeiro Strix hylophyla, MHNCI).

Discussão. Novo registro para o Paraná. Espécie muito semelhante à Icosta americana, pode ser facilmente diferenciada pelos caracteres da chave.

\section{Olfersia Leach, 1817}

Gênero com sete espécies, três restritas ao continente americano; cinco espécies são encontradas sobre aves marinhas (MAA 1969). Apenas duas foram registradas no Paraná.

\section{Chave de identificação para espécies de Olfersia no Paraná, modificada de Bequaert (1957)}

1. Órbita interna com mais de uma fileira de setas (Fig. 8). Órbita interna superior mais curta que o comprimento da órbita interna.... O. bisulcata

$1^{\prime}$. Órbita interna com uma fileira de setas (Fig. 9). Órbita interna superior tão longa quanto o comprimento da órbita interna O. spinifera

\section{Olfersia bisulcata Macquart, 1847}

Distribuição geográfica. Estados Unidos da América, México, Belize, Honduras, Guatemala, Nicarágua, Costa Rica, Panamá, Colômbia, Venezuela, Trinidad e Tobago, Guiana, Guiana Francesa e Brasil (Amazonas, Pará, Piauí, Goiás, Mato Grosso, Mato Grosso do Sul, Minas Gerais, Espírito Santo, Rio de Janeiro, São Paulo e Paraná).

Hospedeiros. Cathartidae (Cathartes Illiger, 1811; Coragyps I. Geoffroy, 1853; Sarcoramphus Duméril, 1806 e Vultur Linnaeus, 1758).

Material examinado. Paraná: 1912 (1 fêmea, hospedeiro Sarcoramphus papa, DZUP).

Discussão. Primeiro registro no estado do Paraná.

\section{Olfersia spinifera (Leach, 1817)}

Distribuição geográfica. Estados Unidos da América, México, Belize, Panamá, Bahamas, Antilhas Maiores, Jamaica, Porto Rico, Saint Croix, Brasil (Ilha de Trindade, Arquipélago de Abrolhos, Rio de Janeiro, São Paulo, Ilha da Queimada, Ilha de Alcatrazes e Paraná), Ilha de Ascension e Equador (Ilhas de Galápagos). Pantropical nos oceanos Pacífico, Atlântico e Índico.

Hospedeiros. Fregatidae (Fregata), Phalacrocoracidae, Sulidae, Pelecanidae e Laridae (MAA 1969).

Material examinado. nenhum.

Discussão. GUIMARÃEs (1945) registrou O. spinifera sobre Fregata magnificens no litoral paranaense.

\section{AGRADECIMENTOS}

À Márcia Árzua, curadora do MHNCI, e aos curadores do DZUP pelo empréstimo de espécimens depositados. A Mauro Pichorim, Cassiano A.F.R. Gatto, Elio C. Guzzo e Maurício O. Moura pela coleta de exemplares utilizados neste trabalho.

\section{REFERÊNCIAS BIBLIOGRÁFICAS}

Bequaert, J. 1942. A monograph of the Melophaginae, or kedflies, of sheep, goats, deer and andantelopes (Diptera, Hippoboscidae). Entomologica Americana, New Series, New York, 22: 173-220.

. 1954. The Hippoboscidae or louse-flies (Diptera) of mammals and birds Part II. Taxonomy, evolution and revision of American genera and species. Entomologica Americana, New Series, New York, 34: 1-232.

. 1955. The Hippoboscidae or louse-flies (Diptera) of mammals and birds Part II. Taxonomy, evolution and revision of American genera and species. Entomologica Americana, New Series, New York, 35: 233-416.

. 1957. The Hippoboscidae or louse-flies (Diptera) of mammals and birds Part II. Taxonomy, evolution and revi- 
sion of American genera and species. Entomologica Americana, New Series, New York, 36: 417-610.

Desiderio, M.H.G.; L. SÁ-Freire; R.H.F. Teixeira; L.C. Cordeiro-Dias \& N.M. Serra-Freire. 1999. Lipoptena (Lipoptenella) guimaraesi Bequaert, 1954 [sic!] (Diptera: Hippoboscidae) em veado campeiro (Ozotocerus bezoarticus Ribeiro, 1919 [sic!]), em Tocantins, Brasil. Entomologia y Vectores, Rio de Janeiro, 16: 204-210.

Grubb, P. 1993. Order Artiodactyla, p. 377-414. In: D.L. WiLson $\&$ D.M. REEDER (Eds). Mammals of the World. A taxonomic and geographic reference. Washington, DC, Smithsonian Institution Press, $2^{\text {nd }}$ ed., XVIII+1206p.

GuimARãES, L.R. 1945. Sobre alguns ectoparasitos de aves e mamíferos do litoral paranaense. Arquivos do Museu Paranaense, Curitiba, 4: 179-190.

JACKSON, J.E. 1987. Ozotoceros bezoarticus. Mammalian species, Northampton, 295: 1-5.

Lutz, A.; A. Neiva \& A. Da Costa Lima. 1915. Sobre "Pupipara" ou "Hippoboscidae" de aves brasileiras. Memórias do Instituto Oswaldo Cruz, Rio de Janeiro, 7: 1-29.
MAA, T.C. 1969. A revised checklist and concise host index of Hippoboscidae (Diptera). Pacific Insects Monographics, Honolulu, 20: 261-299.

. 1989. Family Hippoboscidae, p. 785-789. In: N.L. Evenhuis (Ed.) Catalog of the Diptera of the Australasian and Oceanian Regions. Bishop Museum Special Publication, 86. Bishop Museum Press \& EJ Brill. 1150p.

MaA, T.C. \& B.V. Peterson. 1987. Hippoboscidae, p. 1271-1281. In: J.F. McAlpine, B.V. Peterson, G.E. Shewell, H.J. Tekey, J.R. Vocкевотн \& D.M. Wood (Eds) Manual of Neartic Diptera. Ottawa, Research Branch Agriculture Canada, vol. 2, 1332p.

Peterson, B.V. \& T.C. MaA. 1970. A new Lipoptena from Chile with a key to the New World species (Diptera: Hippoboscidae). The Canadian Entomologist, Ottawa, 102: 1117-1122.

SICK, H. 1997. Ornitologia Brasileira. Rio de Janeiro, Editora Nova Fronteira, 912p.

Sinclair, B.J. 1995. A new species of Ornithoica Rondani from New Caledonia (Diptera: Hippoboscidae). Tijdschrift voor Entomologie, Amsterdam, 140: 129-132.

Recebido em 22.IV.2003; aceito em 07.XI.2003. 